\title{
On the 1-2-3-conjecture
}

\author{
Akbar Davoodi|' and Behnaz Omoomil| \\ Department of Mathematical Sciences, Isfahan University of Technology, Iran
}

received 22 ${ }^{\text {nd }}$ Aug. 2012, revised $8^{\text {th }}$ Mar. 2014, 12 $2^{\text {th }}$ July 2014, $1^{\text {st }}$ Sep. 2014, accepted 20 ${ }^{\text {th }}$ Dec. 2014.

\begin{abstract}
A $k$-edge-weighting of a graph $G$ is a function $w: E(G) \rightarrow\{1, \ldots, k\}$. An edge-weighting naturally induces a vertex coloring $c$, where for every vertex $v \in V(G), c(v)=\sum_{e \sim v} w(e)$. If the induced coloring $c$ is a proper vertex coloring, then $w$ is called a vertex-coloring $k$-edge-weighting (VC $k$-EW). Karoński et al. (J. Combin. Theory Ser. B, 91 (2004) 151-157) conjectured that every graph admits a VC 3-EW. This conjecture is known as the 1-23 -conjecture. In this paper, first, we study the vertex-coloring edge-weighting of the Cartesian product of graphs. We prove that if the 1-2-3-conjecture holds for two graphs $G$ and $H$, then it also holds for $G \square H$. Also we prove that the Cartesian product of connected bipartite graphs admits a VC 2-EW. Moreover, we present several sufficient conditions for a graph to admit a VC 2-EW. Finally, we explore some bipartite graphs which do not admit a VC 2-EW.
\end{abstract}

MSC: $05 \mathrm{C} 15$

Keywords: Vertex-coloring edge-weighting; 1-2-3-conjecture; Edge weighting; Vertex-coloring 2-edge-weighting.

\section{Introduction}

In this paper, we consider finite and simple graphs. An $r$-vertex coloring $c$ of $G$ is a function $c: V(G) \rightarrow$ $\{1, \ldots, r\}$. The coloring $c$ is called a proper vertex coloring if for every two adjacent vertices $u$ and $v$, $c(u) \neq c(v)$. A graph $G$ is $r$-colorable if $G$ has a proper $r$-vertex coloring.

A $k$-edge-weighting of a graph $G$ is a function $w: E(G) \rightarrow\{1, \ldots, k\}$. An edge-weighting naturally induces a vertex coloring $c$, where for every vertex $v \in V(G), c(v)=\sum_{e \sim v} w(e)$, where $e \sim v$ means that $e$ is an edge incident to vertex $v$. If the induced coloring $c$ is a proper vertex coloring, then $w$ is called a vertex-coloring $k$-edge-weighting (VC $k$-EW). The minimum $k$ which $G$ has a $\mathrm{VC} k$-EW is denoted by $\mu(G)$.

Obviously for a graph $G$ with components $G_{1}, G_{2}, \ldots, G_{t} ; \mu(G)=\max \left\{\mu\left(G_{i}\right): 1 \leq i \leq t\right\}$. Also, note that the vertex-coloring $k$-edge-weighting is defined for graphs without $K_{2}$ as a component. Thus, we consider connected graphs with at least three vertices.

Karoński et al. in (Karoński et al. (2004)) introduced the concept of vertex-coloring $k$-edge-weighting and they proposed the following conjecture.

\footnotetext{
*Email: akbar.davoodiemath.iut.ac.ir.

†Email: bomoomi@cc.iut.ac.ir.
} 
Conjecture 1.1. (Karoński et al. (2004)) (1-2-3-conjecture) For every connected graph $G$ with at least three vertices, $\mu(G) \leq 3$.

Addario-Berry et al. (Addario-Berry et al. (2007)) showed that for every connected graph with at least three vertices, $\mu(G) \leq 30$. Then Addario-Berry et al. (Addario-Berry et al. (2008)) improved the bound to 16. Later, Wang and $\mathrm{Yu}$ (Wang and Yu (2008)) improved this bound to 13. Recently, Kalkowski et al. (Kalkowski et al. (2010)) showed that for every connected graph $G$ with at least three vertices, $\mu(G) \leq 5$.

It is proved that for every 3-colorable graph $G, \mu(G) \leq 3$ (Karoński et al.(2004)). In general the similar fact for 2-colorable graphs is not true. The only known families of bipartite graphs with $\mu(G)=3$ are theta graphs $\theta\left(1,4 k_{2}+1,4 k_{3}+1, \ldots, 4 k_{r}+1\right)$, which are graphs obtained by identifying the end-vertices of $r$ paths of length $4 k_{i}+1$ (Chang et al. (2011)).

In (Addario-Berry et al. (2008) ) Addario-Berry et al. proved that almost all graphs admit a VC 2-EW. Moreover, in (Dudek and Wajc (2011)) it was proved that the problem of existence of a VC 2-EW for a given graph is an NP-complete problem. The problem of classifying graphs which admit a VC 2-EW is an open problem. As a more general problem to VC 2-EW, in (Khatirinejad et al. (2012)) Khatirinejad et al. considered the vertex-coloring $\{a, b\}$-edge-weighting, where $a$ and $b$ are arbitrary real numbers. They explore classes of graphs which admit a vertex-coloring $\{a, b\}$-edge-weighting. Furthermore, among some sufficient conditions for a bipartite graph to admit a $\mathrm{VC}\{a, b\}$-EW, they proposed the following question.

Question 1.2. (Khatirinejad et al. (2012)) Is it true that every bipartite graph except $\theta\left(1,4 k_{2}+1,4 k_{3}+\right.$ $\left.1, \ldots, 4 k_{r}+1\right)$ admits a VC $\{a, b\}$-EW, for some real numbers $a$ and $b$ ?

Toward this problem, Havet et al. proved that every bipartite graph with minimum degree at least 3 has a VC $\{a, b\}$-EW, for some $a$ and $b$ (Havet et al. (2014)).

In this paper, we establish more sufficient conditions for a graph to admit a VC 2-EW, Moreover, we construct infinite family of bipartite graphs which do not admit a $\mathrm{VC}\{a, b\}$-EW. Thus, the answer to the question is negative.

In the following we present some theorems which are necessary to get our main results.

Theorem 1.3. (Chang et al. (2011)) If $G ¥ K_{2}$ is a connected bipartite graph satisfying one of the following conditions, then $\mu(G) \leq 2$.

i) $\delta(G)=1$, where $\delta(G)$ is the minimum degree of $G$.

ii) $G$ has a part with an even number of vertices,

Moreover, in (ii) there exists a VC 2-EW for G such that the color of every vertex in the even part is odd and the colors of other vertices are even.

The notion $N[v]$ denotes the set $N(v) \cup\{v\}=\{u: u \in V(G), u v \in E(G)\} \cup\{v\}$.

Theorem 1.4. (Lu et al. (2011) Let $G \varsubsetneqq K_{2}$ be a connected bipartite graph. If one of the following conditions holds, then $\mu(G) \leq 2$.

i) There exists a vertex $v$ such that $\operatorname{deg}(v) \notin\{\operatorname{deg}(u): u \in N(v)\}$ and $G-N[v]$ is connected.

ii) There exists a vertex $v$ of degree $\delta(G)$ such that $\operatorname{deg}(v) \notin\{\operatorname{deg}(u): u \in N(v)\}$ and $G-v$ is connected.

iii) $G$ is 3-connected. 
Theorem 1.5. (Chang et al. (2011)) Let $P_{n}, C_{n}$ and $K_{n}, n \geq 3$, denote the path, cycle and complete graph with $n$ vertices, respectively. Then

$$
\mu\left(P_{n}\right)=\left\{\begin{array}{ll}
1 & n=3 \\
2 & n \geq 4
\end{array}, \mu\left(C_{n}\right)=\left\{\begin{array}{ll}
2 & n \equiv 0(\bmod 4) \\
3 & \text { otherwise }
\end{array}, \text { and } \mu\left(K_{n}\right)=3\right.\right.
$$

The structure of this paper is as follows. In Section 2, we consider the 1-2-3-conjecture for the Cartesian product of graphs. First, we prove that if the 1-2-3-conjecture holds for two graphs $G$ and $H$, then it also holds for $G \square H$. Then, we show that this fact concludes the trueness of 1-2-3-conjecture for the Cartesian product of some well known families of graphs. Moreover, we prove that the Cartesian product of connected bipartite graphs admits a VC 2-EW. Then, we determine $\mu(G)$ for some well known families of graphs. In Section 3 we present several sufficient conditions for a graph to admit a VC 2-EW. In Section 4. we answer Question 1.2 in the negative by showing a new class of graphs admitting no VC $\{a, b\}$-EW, for every real numbers $a, b$.

\section{Cartesian product of graphs}

The Cartesian product of two graphs $G$ and $H$, written $G \square H$, is the graph with vertex set $V(G) \times V(H)$ specified by putting $(u, v)$ adjacent to $\left(u^{\prime}, v^{\prime}\right)$ if and only if $u=u^{\prime}$ and $v v^{\prime} \in E(H)$, or $v=v^{\prime}$ and $u u^{\prime} \in E(G)$.

The vertex-coloring $\{a, b\}$-edge-weighting of Cartesian product of graphs are studied in (Khatirinejad et al. (2012)) and through several theorems, the following result is obtained.

Theorem 2.1. (Khatirinejad et al. (2012)) If $G$ and $H$ are regular bipartite graphs, then the following graphs admit a VC 2-EW.

- $K_{n} \square H$, if $n \geq 4$;

- $C_{n} \square H$, if $n \geq 4$ and $n \neq 5$;

- $G \square H$.

In this section, we prove that if the 1-2-3-conjecture holds for two graphs $G$ and $H$, then it also holds for $G \square H$. Moreover, we prove that if $G$ and $H$ are bipartite graphs, then $\mu(G \square H) \leq 2$.

Theorem 2.2. For every two graphs $G$ and $H, \mu(G \square H) \leq \max \{\mu(G), \mu(H)\}$.

Proof: Let $k=\max \{\mu(G), \mu(H)\}$ and $w_{1}: E(G) \rightarrow\{1,2, \ldots, k\}, w_{2}: E(H) \rightarrow\{1,2, \ldots, k\}$ be VC $k$-EW for graphs $G$ and $H$, respectively. We define $w: E(G \square H) \rightarrow\{1,2, \ldots, k\}, w\left((u, v)\left(u^{\prime}, v\right)\right)=$ $w_{1}\left(u u^{\prime}\right)$ and $w\left((u, v)\left(u, v^{\prime}\right)\right)=w_{2}\left(v v^{\prime}\right)$, where $u, u^{\prime} \in V(G)$ and $v, v^{\prime} \in V(H)$. It is easy to see that $w$ is a VC $k$-EW for $G \square H . \quad \square$ We need the following lemma to prove our main theorem in this section. In what follows $n(G)$ and $\kappa(G)$ denote the order and the vertex connectivity of $G$, respectively.

Lemma 2.3. (Špacapan (2008)) For every two graphs $G$ and $H$,

$$
\kappa(G \square H)=\min \{\delta(G \square H), \kappa(H) n(G), \kappa(G) n(H)\} .
$$

In the following theorem we prove that the Cartesian product of every two bipartite graphs admits a VC 2-EW. 
Theorem 2.4. If $G$ and $H$ are two connected bipartite graphs and $G \square H \neq K_{2}$, then $\mu(G \square H) \leq 2$.

Proof: It can be seen that $G \square H$ is a connected bipartite graph if and only if $G$ and $H$ are connected bipartite graphs. If $n(G)=n(H)=2$, then $G \square H \cong C_{4}$ and by Theorem 1.5, $\mu(G \square H)=2$. If $n(G), n(H)>2$ and both have a leaf vertex, then by Theorems 1.3 $i$ and 2.2. $\mu(G \square H) \leq 2$, otherwise $\delta(G)+\delta(H) \geq 3$. Hence, by Lemma 2.3, $\kappa(G \square H) \geq 3$ and by Theorem 1.4 $(i i i), \mu(G \square H) \leq 2$.

Now, let $n=n(G)>2$ and $n(H)=2$. Thus, $G \square H \cong G \square K_{2}$. Let $G^{(1)}$ and $G^{(2)}$ be the induced subgraphs representing the first and the second copy of $G$, respectively. To give a VC 2-EW for $G \square K_{2}$, first we assign weight 1 to all the edges in $G^{(1)}$ and weight 2 to all the edges in $G^{(2)}$. We denote the unweighted edge $e$ incident to vertex $u \in V\left(G^{(1)}\right)$ by $e_{u}$. Thus, for every two adjacent vertices $u$ and $v$, where $u \in G^{(1)}$ and $v \in G^{(2)}$, independently to the weight of $e_{u}$, we have $c(u) \neq c(v)$. Now we assign a proper weight to the unweighted edges so that for every $u v \in E\left(G^{(1)}\right) \cup E\left(G^{(2)}\right), c(u) \neq c(v)$. We do this as follows.

Suppose $\left(V_{1}, V_{2}\right)$ is the bipartition of $V(G)$ and define

$$
w\left(e_{u}\right)= \begin{cases}1, & \text { if } u \in V_{1} \text { with } \operatorname{deg}(u) \text { even or } u \in V_{2} \text { with } \operatorname{deg}(u) \text { odd } \\ 2, & \text { if } u \in V_{1} \text { with } \operatorname{deg}(u) \text { odd or } u \in V_{2} \text { with } \operatorname{deg}(u) \text { even. }\end{cases}
$$

Then, for vertex $u$ in the first copy, $\sum_{e \sim u} w(e)$ is odd for $u \in V_{1}$ and is even for $u \in V_{2}$. Also, for vertex $u$ in the second copy, $\sum_{e \sim u} w(e)$ is 0 or $1(\bmod 4)$ for $u \in V_{1}$ and is 2 or $3(\bmod 4)$ for $u \in V_{2}$.

Obviously, for every nontrivial graph $G, \mu(G)=1$ if and only if $G$ has no adjacent vertices with a same degree.

Proposition 2.5. For every two graphs $G$ and $H, \mu(G \square H)=1$ if and only if $\mu(G)=1$ and $\mu(H)=1$.

Proof: By Theorem 2.2, the condition is sufficient. Conversely, if $\mu(G \square H)=1$, then $\operatorname{deg}_{G \square H}(u, v) \neq$ $\operatorname{deg}_{G \square H}\left(u^{\prime}, v\right)$, where $u u^{\prime} \in E(G)$. On the other hand $\operatorname{deg}_{G \square H}(u, v)=\operatorname{deg}_{G}(u)+\operatorname{deg}_{H}(v)$. Therefore, for every edge $u u^{\prime} \in E(G), \operatorname{deg}_{G}(u) \neq \operatorname{deg}_{G}\left(u^{\prime}\right)$. This implies $\mu(G)=1$. Similarly, $\mu(H)=1$.

\section{Graphs with $\mu(G) \leq 2$}

In this section, following to investigating the properties of graphs which admit a VC 2-EW, we present several sufficient conditions for a graph to admit a VC 2-EW. In the following, we consider separable graphs.

Theorem 3.1. Let $G$ be a graph which is the union of two simple connected graphs $G_{1}$ and $G_{2}$ such that $V\left(G_{1}\right) \cap V\left(G_{2}\right)=\{v\}$. If for all $u \in N(v), 2 \operatorname{deg}(u) \leq \operatorname{deg}(v)$ and for $i=1,2, G_{i}$ is a cycle or $\mu\left(G_{i}\right) \leq 2$, then $\mu(G) \leq 2$.

Proof: For each $G_{i}$ which is not a cycle, there is a 2-edge-weighting such that $\sum_{e \sim u_{1}} w(e) \neq \sum_{e \sim u_{2}} w(e)$ whenever $u_{1} u_{2} \in E\left(G_{i}-v\right)$. For the case of a cycle, we can weight the edges starting from one adjacent to $v$ by $2,2,1,1$ periodically, such that the last edge be incident to $v$. Thus, in both cases $\sum_{e \sim u_{1}} w(e) \neq \sum_{e \sim u_{2}} w(e)$ whenever $u_{1} u_{2} \in E\left(G_{i}-v\right)$. Moreover, note that since for all $u \in N(v)$, $\sum_{e \sim u} w(e) \leq 2 \operatorname{deg}(u) \leq \operatorname{deg}(v) \leq \sum_{e \sim v} w(e)$ and there is one edge incident on both vertices $u$ and $v$, $\sum_{e \sim u} w(e) \neq \sum_{e \sim v} w(e)$. 
Corollary 3.2. Let $G$ be a simple connected graph with the blocks $B_{1}, B_{2}, \ldots, B_{r}, r \geq 2$, and cut vertices $v_{1}, v_{2}, \ldots, v_{t}$. If for every $j, 1 \leq j \leq t$, for all $u \in N\left(v_{j}\right) 2 \operatorname{deg}(u) \leq \operatorname{deg}\left(v_{i}\right)$ and for every $i, 1 \leq i \leq r$ $B_{i}$ is a cycle or $\mu\left(B_{i}\right) \leq 2$, then $\mu(G) \leq 2$.

A simple path in graph $G$ is a path $P$ in $G$ which the degree of each vertex of $P$ in $G$ is two except the two end-vertices of $P$. The edge set of $G$ is disjoint union of maximal simple paths. Note that a cycle in a graph $G$ with at most one vertex of degree greater than two in $G$ is considered as a maximal simple path in $G$. In the following theorem we give several sufficient conditions for a graph to admit a VC 2-EW with respect to length of maximal simple paths in $G$.

Theorem 3.3. Let $G$ be a connected graph which is not a cycle. If one of the following conditions holds, then $\mu(G) \leq 2$.

i) $G$ contains no maximal simple path of length $3(\bmod 4)$ and for every edge $e=x y$ which is a maximal simple path of $G, \operatorname{deg}(x) \neq \operatorname{deg}(y)$.

ii) $G$ contains no edge as a maximal simple path.

Proof: In each case, we show that there exist a 2-edge-weighting for maximal simple paths in $G$ which induces a proper coloring of $G$.

i) Starting from an end-edge, we assign the weight 1 and 2 to the edges of maximal simple paths of length $2(\bmod 4)$, according to the pattern $2,2,1,1$, periodically. Also for maximal simple paths of length $0,1(\bmod 4)$, we follow the pattern $2,1,1,2$.

According to the given weighting, for two arbitrary adjacent vertices $u$ and $v$, clearly if the degree of one of these vertices is one, then $c(u) \neq c(v)$. Moreover, if the degrees of both vertices $u$ and $v$ are two, then either $u$ and $v$ are internal vertices of a maximal simple path and $c(u) \neq c(v)$.

Now, assume that $\operatorname{deg}(v) \geq 3$ that means $v$ is end-vertex of a maximal simple path. By the given patterns and by the assumption, every edge incident to $v$ has weight 2 , thus $c(v)=2 \operatorname{deg}(v) \geq 6$. If $u$ is an internal vertex for a maximal simple path, then $c(u) \in\{3,4\}$ and $c(u) \neq c(v)$. If $e=u v$ is a maximal simple path of $G$, then by assumption $c(u)=2 \operatorname{deg}(u) \neq 2 \operatorname{deg}(v)=c(v)$.

ii) In this case, similar to above, we weight the edges of maximal simple paths of length $0,1,3$ $(\bmod 4)$, by the pattern $2,1,1,2$. For maximal simple paths of length $2(\bmod 4)$, we follow the pattern $1,1,2,2$.

With a similar discussion as the above one for adjacent vertices $u$ and $v$ both of degree less than three, we have done. Thus, let $\operatorname{deg}(v) \geq 3$. By the assumption, for every vertex $u \in N(v), \operatorname{deg}(u)=2$, hence $c(u) \in\{2,3,4\}$. Clearly if $\operatorname{deg}(v)>4$ or $c(v)>4$, then $c(u) \neq c(v)$. If $\operatorname{deg}(v)=c(v)=4$, then by the given patterns, $c(x)=2$ for every $x \in N(v)$. Thus, particularly $c(u) \neq c(v)$. If $\operatorname{deg}(v)=3$, then there are two possibilities $c(v)=3$ and $c(v)=4$. If $c(v)=3$, then again by the given patterns, $c(u)=2 \neq c(v)$. For the case $\operatorname{deg}(v)=3$ and $c(v)=4$, one of the edges incident to $v$ belongs to a maximal simple path, say $p_{0}$, has weight 2 and two other edges incident to $v$, have weight 1 . Now, $c(u) \neq c(v)$ unless $u \in p_{0}$ and $p_{0}$ is of length $1(\bmod 4)$. Note that the two other paths incident to $v$, say $p_{1}$ and $p_{2}$, are of length 2 or $3(\bmod 4)$.

If $p_{0}$ is of length $1(\bmod 4)$ and $c(u)=c(v)$ and one of the paths $p_{1}$ or $p_{2}$, say $p_{1}$, is of length 3 $(\bmod 4)$, then by reweighting the edges of $p_{1}$ by the same pattern but starting from $v$, we get $c(v)=5>$ $c(x)$ for every vertex $x \in N(v)$. In this process no new adjacent vertices with a same color occurs.

If $u \in p_{0}, p_{0}$ is of length $1(\bmod 4), c(u)=c(v)$ and both $p_{1}$ and $p_{2}$ are of length $2(\bmod 4)$, then we reweight the edges of $p_{0}$ by the same pattern as before but starting from $v$, thus we get $c(v) \neq c(x)$ for 
every vertex $x \in N(v)$. We have no new adjacent vertices with the same color, unless the other end-vertex of $p_{0}$, say $v^{\prime}$, has the conditions exactly the same as $v$. That means $\operatorname{deg}\left(v^{\prime}\right)=3$ and $c\left(v^{\prime}\right)=4$. By the same argument, we solve the problem when at least one of the paths incident to $v^{\prime}$ is of length $3(\bmod 4)$.

Otherwise, both of the other paths incident to $v^{\prime}$ are of length $2(\bmod 4)$. In this case we choose one of them and change its edge weight pattern from $1,1,2,2$ to $2,2,1,1$. If the other end of this path, say $v^{\prime \prime}$, is not of color 4 or is not of degree 3 , we have done as above. Otherwise we do this process for $v^{\prime \prime}$.

Since the graph is finite, after iterating this process we face to an end-vertex of a maximal simple path for the second time. While the color of this vertex, after changing the weights according to the given pattern in the second visit is at least five. Thus, this process terminates and in this manner, we reduce the number of adjacent vertices with the same color to get the desired weighting.

$\square$ For the

case that $G$ contains an edge as a maximal simple path there are graphs with $\mu(G)=2$ and $\mu(G)=3$. The complete graph $K_{n}$ is an example of graphs in which every maximal simple path is of length 1 and $\mu\left(K_{n}\right)=3$. The following proposition gives an example of graphs in which every maximal simple path is of length 1 and $\mu(G)=2$.

Proposition 3.4. For every complete $r$-partite graph $K_{r * n}, r, n \geq 2, \mu\left(K_{r * n}\right)=2$.

Proof: Let $A$ be a weighted adjacency matrix of $G=K_{r * n}$, defined as follows.

$$
A=\left(\begin{array}{cccc}
0 & B & \cdots & B \\
B^{T} & \ddots & \ddots & \vdots \\
\vdots & \ddots & \ddots & B \\
B^{T} & \cdots & B^{T} & 0
\end{array}\right)_{r n \times r n} \text {, where } B=\left(\begin{array}{cccc}
1 & 1 & \ldots & 1 \\
\vdots & \vdots & & \vdots \\
1 & 1 & \ldots & 1 \\
2 & 2 & \ldots & 2
\end{array}\right)_{n \times n}
$$

Note that the induced color on every vertex is the sum of entries in it's corresponding row in $A$. Thus, for every two arbitrary vertices $u$ and $v$ in $i$-th part and $j$-th part of $G, c(u) \in\{(r-i) n+(i-1)(n+$ 1), $2(r-i) n+(i-1)(n+1)\}$ and $c(v) \in\{(r-j) n+(j-1)(n+1), 2(r-j) n+(j-1)(n+1)\}$. Since $i \neq j$ we have

$$
(r-i) n+(i-1)(n+1) \neq(r-j) n+(j-1)(n+1)
$$

and

$$
2(r-i) n+(i-1)(n+1) \neq 2(r-j) n+(j-1)(n+1)
$$

On the other hand, $j \leq r$ hence, $j-i \leq n(r-i)$ with equality if and only if $n=1$ and $j=r$. Thus $(r-j) n+(j-1)(n+1) \neq 2(r-i) n+(i-1)(n+1)$. Similarly $(r-i) n+(i-1)(n+1) \neq$ $2(r-j) n+(j-1)(n+1)$. Therefore, $A$ is a desired edge-weighting. $\square$ We end this section with the following conjecture.

Conjecture 3.5. Let $G \nsubseteq C_{4 k+r}, r \in\{1,2,3\}$, be a connected graph. If $\mu(G)>2$, then $G$ has two adjacent vertices of a same degree greater than two. 


\section{Bipartite graphs}

It is known that every 3-colorable graph admits a VC 3-EW (Karoński et al. (2004)), and there are bipartite graph $G$ with $\mu(G)=2$ and also bipartite graph $G$ with $\mu(G)=3$. The only known bipartite graphs with $\mu(G)=3$ are $C_{4 k+2}$ and the theta graphs $\theta\left(1,4 k_{2}+1, \ldots, 4 k_{r}+1\right)$ (Chang et al. (2011)). The theta graph $\theta\left(l_{1}, l_{2}, \ldots, l_{r}\right)$ is the graph obtains from $r$ disjoint paths, of lengths $l_{1}, l_{2}, \ldots, l_{r}$, respectively, by identifying their end-vertices called the roots of the graph. Notice that $\theta\left(l_{1}\right)=P_{1+l_{1}}$ and $\theta\left(l_{1}, l_{2}\right)=$ $C_{l_{1}+l_{2}}$.

Theorem 4.1. (Chang et al. (2011)) Let $G=\theta\left(l_{1}, l_{2}, \ldots, l_{r}\right)$, where $r \geq 3$ and $1 \leq l_{1} \leq l_{2} \leq \ldots \leq l_{r}$. Then,

$$
\mu(G)= \begin{cases}1 & l_{i}=2 \text { for all } i \\ 3 & l_{1}=1 \text { and } l_{i} \equiv 1(\bmod 4) \text { for all } i \neq 1 \\ 2 & \text { otherwise }\end{cases}
$$

In this section, we consider a generalization of theta graphs, called generalized polygon trees, and answer to Question 1.2 in negative.

The class of generalized polygon trees is defined recursively as follows. A cycle $C_{p}(p \geq 3)$ is a generalized polygon tree. Next, suppose $H$ is a generalized polygon tree containing a simple path $P_{k}$, where $k \geq 1$. The graph obtained from the disjoint union of $H$ and a cycle $C_{r}$, where $r>k$, by identifying $P_{k}$ in $H$ with a path of length $k$ in $C_{r}$, is also a generalized polygon tree.(Dong et al. (2005))

In Theorem 4.1, $\mu(G)$ is determined whenever $G$ is a generalized polygon tree with two interior regions. In this section, we determine the $\mu(G)$ for bipartite generalized polygon trees with three interior regions (see Figure 1).

We need the following sufficient condition to guarantee that a bipartite graph admits a VC 2-EW.

Theorem 4.2. Let $G$ be a connected bipartite graph. If $G$ contains a vertex $v$ such that $\operatorname{deg}(v)>\operatorname{deg}(u)$ for every $u \in N(v)$ and $G-v$ is connected, then $\mu(G) \leq 2$.

Proof: Let $U$ and $W$ be the parts of $G$. If either $|U|$ or $|W|$ is even, then by Theorem $1.3(i i)$, the result follows. Thus, we assume that both parts of $G$ are of odd size. Let $v \in U$ satisfy $\operatorname{deg}(v)>\operatorname{deg}(u)$ for every $u \in N(v)$ and $G-v$ is connected. Since $|U-v|$ is even, by Theorem $1.3(i i), G-v$ admits a VC 2-EW such that $c(x)$ is odd for every $x \in U-v$ and $c(y)$ is even for every $y \in W$. Now we assign weight 2 to all the edges that are adjacent to vertex $v$. Clearly $c(x)$ is odd for every $x \in U-v$ and $c(y)$ is even for every $y \in W$. Also $c(v)=2 \operatorname{deg}_{G}(v)>2 \operatorname{deg}_{G}(u) \geq c(u)$ for every $u \in N(v)$.

Fact 4.3. In every VC 2-EW of a path, the weight of one edge forces the weights of every second edge. Thus, if $P$ is a path of length $l, l \equiv 1(\bmod 4)$, then two end-edges of $P$ get a same weight and if $l \equiv 3(\bmod 4)$, then they get different weights. Furthermore, when $l \neq \equiv 1,3(\bmod 4)$ every arbitrary assignment of the end-edges of $P$ with $\{1,2\}$, could be extended to a VC 2-EW for $P$.

Theorem 4.4. Let $G$ be a bipartite generalized polygon tree with at most three interior regions shown in Figure 1 where $p_{1}, p_{2}, \ldots, p_{6}$ are paths of length $a, b, c, d$, e and $f$, respectively. Then $\mu(G)=3$ if and only if one of the following conditions holds.

- $a=b=c=d=0$ and $e+f \equiv 2(\bmod 4)$.

- $b=1, c=d=0$ and $a, e+f \equiv 1(\bmod 4)$. 
- $b=1, e=f=0$ and $a, c, d \equiv 1(\bmod 4)$.

- $b=c=1, a, d, e \equiv 1(\bmod 4)$ and $f \equiv 3(\bmod 4)$.

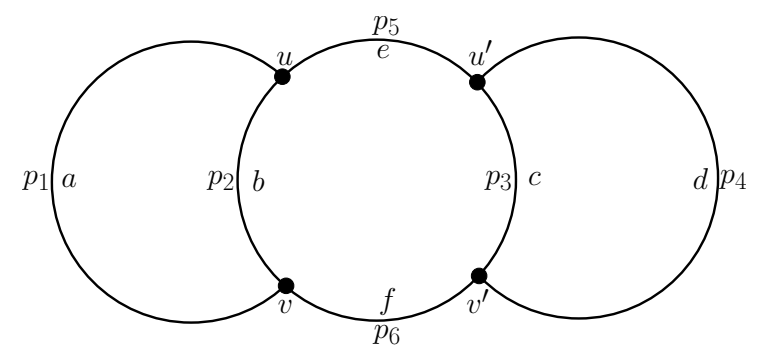

Fig. 1: A generalized polygon tree with three interior regions.

Proof: We know that a graph admits a vertex-coloring 1-edge-weighting if and only if every two adjacent vertices have different degrees. It can be verified that this condition holds if and only if $a=b=c=d=$ $e=f=2$ or $a=d=f=2, b=c=1$ and $e=0$ or $a=d=f=2, b=c=2$ and $e=0$ or $a=b=c=d=2$ and $e=f=0$.

For $b$ and $c$ there are three possibilities $b=c=0$ or $b \neq 0, c=0$ or $b, c \neq 0$.

Let $b=c=0$. If $a=d=0$, then $G$ is an even cycle $C_{e+f}$ and by Theorem 1.5 if $e+f \equiv 0(\bmod 4)$, then $\mu(G)=2$, otherwise, $\mu(G)=3$. If $a \neq 0$ or $d \neq 0$, by Corollary 3.2, $\mu(G)=2$.

Now let $b \neq 0$ and $c=0$. Since $d \neq 0, G$ is a graph with two blocks: a $\theta(a, b, e+f)$ and a cycle $C_{d}$. Thus by Theorem 3.1. $\mu(G)=2$ unless $\mu(\theta(a, b, e+f))=3$. In this case we give a VC 2 -EW for $G$ in Figure 22a. In the figures the edges and paths are denoted by straight lines and curves, respectively, and every path is weighted periodically by the given pattern through the denoted direction.

Otherwise $b, c \neq 0$. In this case, we consider three possibilities $b, c>1$ or $b=1, c>1$ or $b=c=1$.

Now let $b, c>1$. In this case if $e \neq 1$ or $f \neq 1$, then there exists a vertex $x$ with $\operatorname{deg}(x) \geq 3$ such that $\operatorname{deg}(x)>\operatorname{deg}(y)$ for all $y \in N(x)$ and $G-x$ is connected. Thus by Theorem $4.2 \mu(G) \leq 2$. Therefore we assume that $e=f=1$. Notice that if $a=0$ or $d=0$, then $G$ is a theta graph and we have done. Now, since $G$ is bipartite $b+c$ is even. Hence, $b+c \equiv 0(\bmod 4)$ or $b+c \equiv 2(\bmod 4)$. For the first possibility if there is a part of even order, by Theorem $1.3(i i)$, we have done. Otherwise we have two odd parts and $a+d \equiv 2(\bmod 4)$. In this case we have four possibilities $a \equiv 2(\bmod 4), b, c, d \equiv 0$ $(\bmod 4)$ or $a, b, c \equiv 2(\bmod 4), d \equiv 0(\bmod 4)$ or $a, b, d \equiv 1(\bmod 4), c \equiv 3(\bmod 4)$ or $a, c, d \equiv 3$ $(\bmod 4), b \equiv 1(\bmod 4)$, in which in each case the given pattern in Figure $2 \mathrm{~b}$ is a VC 2 -EW for $G$. For the latter case, $a+d \equiv 2(\bmod 4)$ give a part with even number of vertices and if both parts have odd number of vertices and $a+d \equiv 0(\bmod 4)$ by the symmetry of $b, c$ and $a, d$ with the same discussion we get the desired result.

Now let $b=1, c>1$. If $e=f=0$, then $G \cong \theta(a, 1, c, d)$. Thus, we assume that $\{e, f\} \neq\{0\}$. In this case if $e \neq 1$ or $f \neq 1$, then there exists a vertex $x$ with $\operatorname{deg}(x) \geq 3$ such that $\operatorname{deg}(x)>\operatorname{deg}(y)$ for all $y \in N(x)$ and $G-x$ is connected. Thus by Theorem $4.2, \mu(G) \leq 2$. Therefore, we assume $e=f=1$. Since $G$ is bipartite $a, c$ and $d$ are odd. For the cases $a, c, d \equiv 3(\bmod 4)$ and $a \equiv 3(\bmod 4), c, d \equiv 1$ $(\bmod 4)$ and $a, c \equiv 1(\bmod 4), d \equiv 3(\bmod 4)$ in Figure $2 \mathrm{c}$ is given a VC 2 -EW. Otherwise, $G$ has a part of even order and by Theorem 1.3 ii $), \mu(G) \leq 2$. 


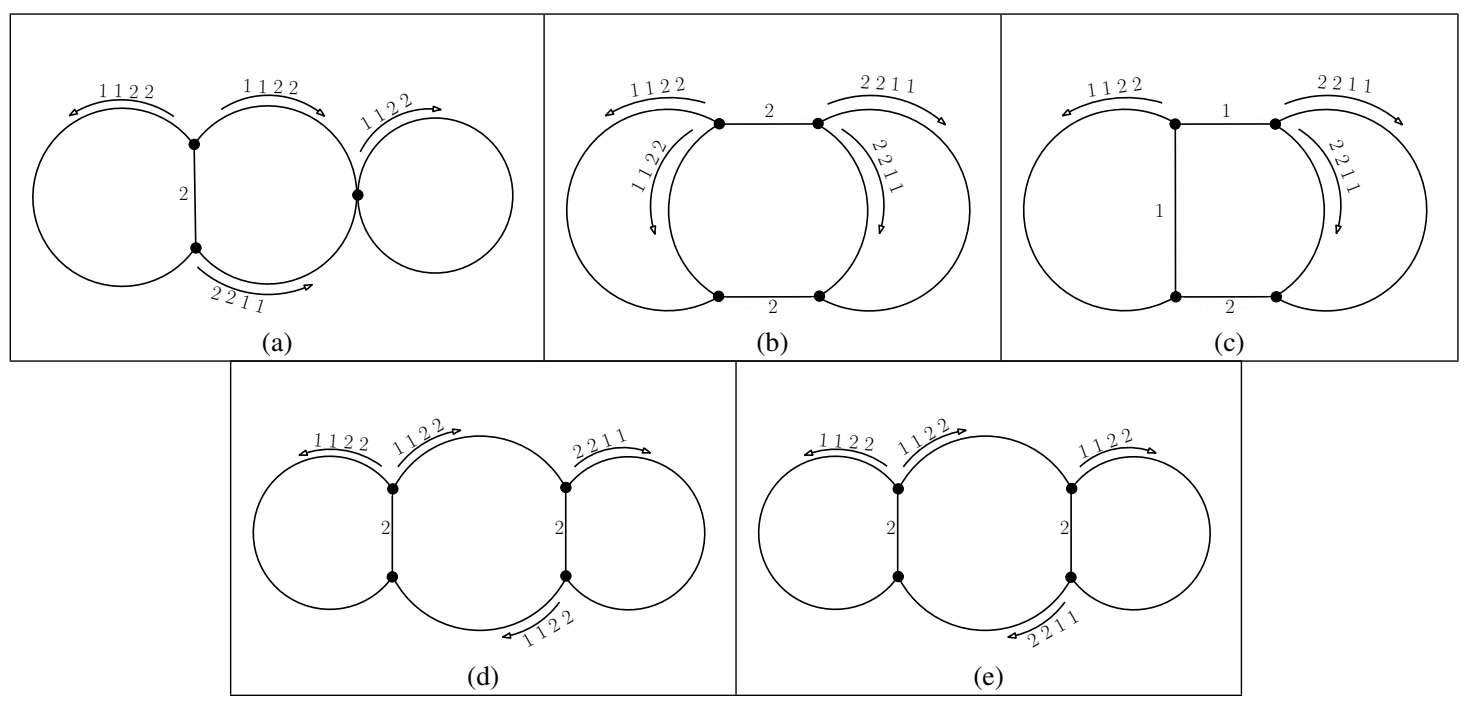

Fig. 2: A VC 2-EW for $G$.

Now if $b=c=1$, then since $G$ is bipartite, $e+f$ is even and $a, d$ are odd.

Case 1. $a, d \equiv 1(\bmod 4)$. If $e+f \equiv 2(\bmod 4)$, then $G$ has a part of even number of vertices and by Theorem $1.3(i i), \mu(G) \leq 2$. Thus, we assume $e+f \equiv 0(\bmod 4)$. For the cases $e, f \equiv 0(\bmod 4)$ and $e, f \equiv 2(\bmod 4)$ we give a VC 2-EW for $G$ in Figures $2 \mathrm{~d}$ and $2 \mathrm{e}$, respectively.

For the case $e \equiv 1, f \equiv 3(\bmod 4)$ first we show that $\mu(G) \geq 3$. Suppose to the contrary that $G$ admits a VC 2-EW. Since $a \equiv 1(\bmod 4)$, by Fact 4.3 , in $p_{1}$ the incidence edges on $u$ and $v$ have a same weight. Similarly incidence edges on $u^{\prime}$ and $v^{\prime}$ in $p_{4}$. Thus, the incidence edges on $u, v$ through $p_{5}$ and $p_{6}$ must have different weights. On the other hand, since $e \equiv 1(\bmod 4)$, two end-edges on $p_{5}$ get the same weight but two edges on $p_{6}$ get different weights, because $f \equiv 3(\bmod 4)$. Therefore $c\left(u^{\prime}\right)=c\left(v^{\prime}\right)$. This contradiction implies $\mu(G) \geq 3$. On the other hand, $G$ is bipartite, thus is 3-colorable and $\mu(G) \leq 3$. Therefore, $\mu(G)=3$.

Case 2. $a, d \equiv 3(\bmod 4)$. If $e+f \equiv 2(\bmod 4)$, then by Theorem $1.3(i i), \mu(G) \leq 2$. Thus, we assume $e+f \equiv 0(\bmod 4)$. Now one of the three possibilities $e, f \equiv 0(\bmod 4)$ or $e, f \equiv 2(\bmod 4)$ or $e \equiv 1, f \equiv 3(\bmod 4)$ occurs, in which in each case the given pattern in Figure 2d is a VC 2-EW for $G$.

Case 3. $a \equiv 1, d \equiv 3(\bmod 4)$. If $e+f \equiv 0(\bmod 4)$, then by Theorem $1.3(i i), \mu(G) \leq 2$. Thus, we assume $e+f \equiv 2(\bmod 4)$ and for the cases $e, f \equiv 1(\bmod 4)$ or $e \equiv 0(\bmod 4), f \equiv 2(\bmod 4)$ and the case $e, f \equiv 3(\bmod 4)$ we give a VC 2 -EW for $G$ in Figures $2 \mathrm{e}$ and $2 \mathrm{~d}$ respectively. Notice that in the case $e \equiv 0(\bmod 4), f \equiv 2(\bmod 4)$ if $e=0$, then by Theorem $4.2, \mu(G) \leq 2$. Also in the case $e, f \equiv 1(\bmod 4)$ if $e=1$, we can replace weight of the edge $u v$ with 1 to get a VC 2-EW. 
In Theorem 4.4 we obtained infinite classes of bipartite graphs with $\mu(G)=3$. It can be seen that the first three cases of such graphs are $C_{4 k+2}, \theta\left(1,4 k_{2}+1,4 k_{3}+1\right)$ and $\theta\left(1,4 k_{2}+1,4 k_{3}+1,4 k_{4}+1\right)$, respectively. Furthermore, The graphs satisfying the fourth condition can be generalize to the following example which provides more bipartite graphs with $\mu(G)=3$.

Remark 4.5. Fact 4.3 is true the edges are weighted with every two arbitrary real numbers $a$ and $b$. Thus, the proof of Theorem 4.4 can be applied to characterize generalized polygon trees with at most three interior regions that has no VC $\{a, b\}$-EW.

Example 4.6. Let $G$ be a graph shown in Figure 3 in which $Q_{i}, 1 \leq i \leq n+1$, are paths of length 1 $(\bmod 4)$ and $R_{i}, S_{i}, 1 \leq i \leq n$, are paths of odd lengths $r_{i}, s_{i}$, where $r_{i}+s_{i} \equiv 0(\bmod 4)$. By Remark 4.5 , it is easy to see that $G$ has no VC $\{a, b\}$-EW. Thus since $G$ is bipartite, $\mu(G)=3$.

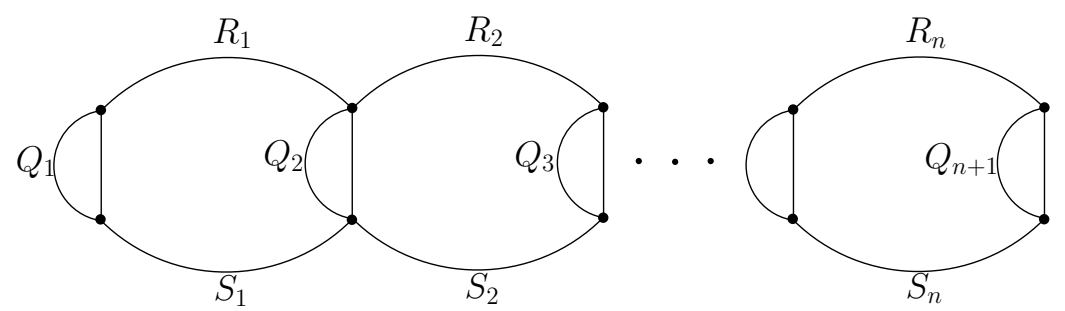

Fig. 3: A family of 2-connected bipartite graphs with no VC $\{a, b\}$-EW.

Note that, we can add any number of paths of length $1(\bmod 4)$ to $G$ in Figure 3 with the same end-vertices with $Q_{i}$ to get more bipartite graphs with no $\mathrm{VC}\{a, b\}$-EW. Also, the graphs described in Example 4.6 can be generalized to a family of graphs as follows.

Consider the family $\mathcal{F}$ of graphs recursively defined by the following two rules.

(i) The family $\mathcal{F}$ contains $\left(C_{4 k+2} ; M\right)$, where $M$ is a perfect matching in $C_{4 k+2}$.

(ii) If $(G ; M) \in \mathcal{F}$, then $\left(G^{\prime} ; M^{\prime}\right) \in \mathcal{F}$, where $G^{\prime}$ is obtained from a path $v_{0}, v_{1}, v_{2}, \ldots, v_{4 r+1}$ disjoint from $G$ by identifying $v_{0}$ with $x$ and $v_{4 r+1}$ with $y$ for some edge $x y \in M$, and $M^{\prime}=M \cup\left\{v_{2 i-1} v_{2 i}\right.$ : $1 \leq i \leq 2 r\}$. Notice that the graphs in Theorem 4.4 are all in this family.

Theorem 4.7. For any $(G ; M) \in \mathcal{F}$ and any two distinct real numbers a and $b$, the graph $G$ has no $V C$ $\{a, b\}-E W$.

Proof: First note that for any real numbers $a$ and $b, C_{4 k+2}$ has no VC $\{a, b\}$-EW. Now, by the contrary assume that $\left(G^{\prime} ; M^{\prime}\right)$ is a graph in $\mathcal{F}$ and for some real numbers $a$ and $b, G^{\prime}$ has a $\operatorname{VC}\{a, b\}$-EW, say $w^{\prime}$. Now, let $H=\theta\left(1,4 k_{1}+1,4 k_{2}+1, \ldots, 4 k_{t}+1\right), t \geq 1$, be an induced subgraph of $G^{\prime}$ with roots $x$ and $y$, for some adjacent vertices $x$ and $y$, where $\operatorname{deg}_{H}(x)=\operatorname{deg}_{G^{\prime}}(x)-1$ and $\operatorname{deg}_{H}(y)=\operatorname{deg}_{G^{\prime}}(y)-1$ (note that since $G^{\prime} \neq C_{4 k+2}$, by the construction of $\mathcal{F}$ such a subgraph has always exists). Thus, $G^{\prime}$ is obtained by identifying the two end vertices of paths $P_{1}: v_{0}=x, v_{1}, v_{2}, \cdots, v_{4 k_{1}+1}=y, P_{2}, \ldots, P_{t}$ in $H$ with the vertices $x$ and $y$ of graph $(G, M) \in \mathcal{F}$, where $G=\left(G^{\prime} \backslash E(H)\right) \cup\{x y\}$ and $x y \in M$.

In the following, we provide a $\mathrm{VC}\{a, b\}$-EW for $G$. Let $w$ be the restriction of $w^{\prime}$ to $E(G)$ and $c(v)=\sum_{e \sim v} w(e)$, for all $v \in V(G)$. Thus, $c(u) \neq c(v)$, for all $u, v \in V(G) \backslash\{x, y\}$, also by Remark $4.5 . c(x) \neq c(y)$. If $w$ is a $\operatorname{VC}\{a, b\}$-EW for $G$, then we are done. Otherwise, there is a unique adjacent vertex to $x$ in $G$, say $x_{1}$ (resp. to $y$ in $G$, say $y_{1}$ ) that may get the color $c(x)$ (resp. $c(y)$ ). 
Let $d:=c^{\prime}(x)-c(x)$, where $c^{\prime}$ and $c$ are the colorings obtained by $w^{\prime}$ and $w$, respectively. Note that by Remark 4.5. for each $P_{i}, 1 \leq i \leq t$, the two end edges of $P_{i}$ have a same weight. Now, if $w^{\prime}\left(x v_{1}\right)=a$ or $w^{\prime}\left(x v_{1}\right)=b$, then $d=\alpha+a$ or $d=\alpha+b$, respectively, where $\alpha$ is the sum of the weight of edges incident on $x$ in $\cup_{i=2}^{t} P_{i}$. Moreover, if $w^{\prime}\left(x v_{1}\right)=a$ and $w^{\prime}(x y)=a$, then by changing $w(x y)$ to $b$, we have $d=\alpha+2 a-b$. Similarly, if $w^{\prime}\left(x v_{1}\right)=b$ and $w^{\prime}(x y)=b$, then by changing $w(x y)$ to $b$, we have $d=\alpha+2 b-a$. Therefore, by changing the weight of the edge $x y$ (if it is necessary), all of the four distinct values $\alpha+a, \alpha+b, \alpha+2 a-b, \alpha+2 b-a$ can be obtained (It should be note that each of the values $a$ and $b$ can be supposed to be assigned to the edge $x v_{1}$ in $\left.w^{\prime}\right)$. Thus, there exists $d \neq 0$ such that $d \neq c^{\prime}(x)-c^{\prime}\left(x_{1}\right)$ and $d \neq c^{\prime}(y)-c^{\prime}\left(y_{1}\right)$. Since $c^{\prime}(x)-d=c(x)$ and $c^{\prime}(y)-d=c(y)$, we have $c(x) \neq c\left(x_{1}\right)$ and $c(y) \neq c\left(y_{1}\right)$. Consequently, we could get a VC $\{a, b\}$-EW for $G$. Continuing this procedure leads us to a VC $\{a, b\}$-EW for $C_{4 k+2}$ which is a contradiction.

The structures of known bipartite graphs with no $\mathrm{VC}\{a, b\}$-EW, encourage us to ask the following question.

Question 4.8. Is it true that every bipartite graph does not belong to the family $\mathcal{F}$ admits a $\operatorname{VC}\{a, b\}$-EW, for some real numbers $a$ and $b$ ?

\section{Acknowledgement}

The authors would like to thank the referees for their valuable comments and introduce the family $\mathcal{F}$ in Theorem 4.7

\section{References}

L. Addario-Berry, K. Dalal, C. McDiarmid, B. A. Reed, and A. Thomason. Vertex-colouring edgeweightings. Combinatorica, 27(1):1-12, 2007. ISSN 0209-9683. doi: 10.1007/s00493-007-0041-6. URL http://dx.doi.org/10.1007/s00493-007-0041-6

L. Addario-Berry, K. Dalal, and B. A. Reed. Degree constrained subgraphs. Discrete Appl. Math., 156 (7):1168-1174, 2008. ISSN 0166-218X. doi: 10.1016/j.dam.2007.05.059. URL http://dx.doi. org/10.1016/j.dam.2007.05.059.

G. J. Chang, C. Lu, J. Wu, and Q. Yu. Vertex-coloring edge-weightings of graphs. Taiwanese J. Math., 15(4):1807-1813, 2011. ISSN 1027-5487.

F. M. Dong, K. M. Koh, and K. L. Teo. Chromatic polynomials and chromaticity of graphs. World Scientific Publishing Co. Pte. Ltd., Hackensack, NJ, 2005. ISBN 981-256-383-0. doi: 10.1142/ 9789812569462. URL http://dx.doi.org/10.1142/9789812569462

A. Dudek and D. Wajc. On the complexity of vertex-coloring edge-weightings. Discrete Math. Theor. Comput. Sci., 13(3):45-50, 2011. ISSN 1365-8050.

F. Havet, N. Paramaguru, and R. Sampathkumar. Detection number of bipartite graphs and cubic graphs. Discrete Math. Theor. Comput. Sci., 16(3):333-342, 2014. 
M. Kalkowski, M. Karoński, and F. Pfender. Vertex-coloring edge-weightings: towards the 1-2-3conjecture. J. Combin. Theory Ser. B, 100(3):347-349, 2010. ISSN 0095-8956. doi: 10.1016/j.jctb. 2009.06.002. URL http://dx.doi.org/10.1016/j.jctb.2009.06.002

M. Karoński, T. Łuczak, and A. Thomason. Edge weights and vertex colours. J. Combin. Theory Ser. B, 91(1):151-157, 2004. ISSN 0095-8956. doi: 10.1016/j.jctb.2003.12.001. URL http://dx.doi. org/10.1016/j.jctb.2003.12.001.

M. Khatirinejad, R. Naserasr, M. Newman, B. Seamone, and B. Stevens. Vertex-colouring edgeweightings with two edge weights. Discrete Math. Theor. Comput. Sci., 14(1):1-20, 2012. ISSN $1365-8050$.

H. Lu, Q. Yu, and C.-Q. Zhang. Vertex-coloring 2-edge-weighting of graphs. European J. Combin., 32 (1):21-27, 2011. ISSN 0195-6698. doi: 10.1016/j.ejc.2010.08.002. URL http://dx.doi.org/ $10.1016 / j . e j c .2010 .08 .002$.

S. Špacapan. Connectivity of Cartesian products of graphs. Appl. Math. Lett., 21(7):682-685, 2008. ISSN 0893-9659. doi: 10.1016/j.aml.2007.06.010. URL http://dx.doi.org/10.1016/j. aml.2007.06.010.

T. Wang and Q. Yu. On vertex-coloring 13-edge-weighting. Front. Math. China, 3(4):581-587, 2008. ISSN 1673-3452. doi: 10.1007/s11464-008-0041-x. URL http://dx.doi.org/10.1007/ s11464-008-0041-x. 